\title{
Role of $U c p 1$ enhancer methylation and chromatin remodelling in the control of $U c p 1$ expression in murine adipose tissue
}

\author{
A. Shore • A. Karamitri • P. Kemp • J. R. Speakman • \\ M. A. Lomax
}

Received: 13 November 2009 / Accepted: 19 January 2010/Published online: 18 March 2010

(C) The Author(s) 2010. This article is published with open access at Springerlink.com

\begin{abstract}
Aims/hypothesis Increasing the expression of the brown adipose tissue-specific gene uncoupling protein-1 (Ucp1) is a potential target for treating obesity. We investigated the role of DNA methylation and histone modification in Ucp 1 expression in adipose cell lines and ex vivo murine adipose tissues.

Methods Methylation state of the Ucp1 enhancer was studied using bisulphite mapping in murine adipose cell lines, and tissue taken from cold-stressed mice, coupled with functional assays of the effects of methylation and
\end{abstract}

Electronic supplementary material The online version of this article (doi:10.1007/s00125-010-1701-4) contains supplementary material, which is available to authorised users.

A. Shore

Division of Biomedical Science, Imperial College, Wye Campus, Ashford, Kent, UK

A. Karamitri $\cdot$ M. A. Lomax $(\bowtie)$

School of Biosciences, Division of Nutritional Sciences,

University of Nottingham,

Sutton Bonington Campus,

Loughborough, Leicestershire LE12 5RD, UK

e-mail: michael.lomax@nottingham.ac.uk

\section{P. Kemp}

National Heart and Lung Institute, Faculty of Medicine, Imperial College London,

London, UK

J. R. Speakman

School of Biological Sciences, University of Aberdeen,

Aberdeen, UK

Present Address:

A. Shore

School of Biosciences, Cardiff University,

Cardiff, UK demethylation of the Ucpl promoter on gene expression and nuclear protein binding.

Results We show that demethylation of the Ucpl promoter by 5-aza-deoxycytidine increases Ucpl expression while methylation of Ucp 1 promoter-reporter constructs decreases expression. Brown adipose tissue-specific Ucp 1 expression is associated with decreased $\mathrm{CpG}$ dinucleotide methylation of the Ucpl enhancer. The lowest $\mathrm{CpG}$ dinucleotide methylation state was found in two cyclic AMP response elements (CRE3, CRE2) in the Ucp1 promoter and methylation of the $\mathrm{CpG}$ in CRE2, but not CRE3 decreased nuclear protein binding. Chromatin immunoprecipitation assays revealed the presence of the silencing DiMethH3K9 modification on the Ucpl enhancer in white adipose tissue and the appearance of the active TriMethH3K4 mark at the Ucp 1 promoter in brown adipose tissue in response to a cold environment.

Conclusions/interpretation The results demonstrate that $\mathrm{CpG}$ dinucleotide methylation of the Ucpl enhancer exhibits tissue-specific patterns in murine tissue and cell lines and suggest that adipose tissue-specific Ucp 1 expression involves demethylation of $\mathrm{CpG}$ dinucleotides found in regulatory CREs in the Ucpl enhancer, as well as modification of histone tails.

Keywords $\mathrm{CpG}$ dinucleotide - Methylation - Cyclic AMP response element - Uncoupling protein-1 - Adipose tissue

$\begin{array}{ll}\text { Abbreviations } \\ \text { BAT } & \text { Brown adipose tissue } \\ \text { ChIP } & \text { Chromatin immunoprecipitation } \\ \text { CRE } & \begin{array}{l}\text { Cyclic adenosine monophosphate response } \\ \text { element }\end{array} \\ \text { CREB } & \text { CRE-binding protein } \\ \text { EMSA } & \text { Electrophoretic mobility shift assays }\end{array}$


gWAT Gonadal white adipose tissue

iBAT Intrascapular brown adipose tissue

iWAT Intrascapular white adipose tissue

PGC-1 Peroxisome proliferator activated receptor gamma coactivator 1

PPARE Peroxisome proliferator activated receptor response element

qRTPCR Quantitative real-time PCR

WAT White adipose tissue

\section{Introduction}

Obesity is a major risk factor for the development of diabetes and cardiovascular disease. Treatments for obesity have centred on decreasing appetite rather than increasing energy expenditure because humans proved to be refractory to $\beta$-adrenergic agonists aimed at inducing an increase in brown adipose tissue (BAT) thermogenesis. This was despite promising work in rodents that energy expenditure can be increased by $\beta$-selective adrenergic stimulation of the brown fat thermogenic gene, uncoupling protein 1 (Ucp1) [1]. Recently there has been renewed interest in the role of BAT in humans since fluorodeoxyglucose positron emission tomography has revealed the presence of BAT depots in adult humans [2]. Furthermore, human white adipocytes can acquire the molecular features of brown adipocytes [3]. Therefore, increasing the numbers of brown adipocytes in humans has been suggested to be a potential target for treating obesity $[4,5]$.

We have demonstrated that suppressed adrenergicsensitive Ucpl expression in the white adipocyte 3T3-L1 cell line is not the result of inhibition of adrenergic signalling, and can be stimulated by enhancing transcriptional activity from the cyclic AMP response elements (CREs) in BAT genes [4, 5]. Our earlier studies on the ontogenic development of BAT in sheep, suggested that this species was similar to humans in that adrenergic-sensitive Ucp1 expression was suppressed soon after birth [6]. Methylation of $\mathrm{CpG}$ dinucleotides in gene promoters is thought to play a key role during the developmental control of cell-specific gene expression in association with histone tail modification to regulate chromatin structure and function [7]. These data suggested to us that gene silencing mechanisms involving DNA methylation of CREs and chromatin remodelling may be important in regulating Ucp1 expression.

BAT is characterised by large numbers of mitochondria, increased fatty acid oxidation and a capacity for high metabolic rate due to the action of $U c p 1$, which uncouples oxidative phosphorylation [8]. Ucpl expression is BATspecific and is regulated by a basal promoter $\sim 250$ bp upstream from the start of transcription, a silencer unit at $-1000 \mathrm{~kb}$, and a highly conserved $221 \mathrm{bp}$ enhancer element located approximately $-2.5 \mathrm{~kb}$ from the transcriptional start site in the mouse and rat promoters and $-3.9 \mathrm{~kb}$ in the human promoter. A number of binding sites for nuclear receptors and bZIP transcriptional factors are located in the enhancer region and have been shown to play functional roles in the stimulation of $U c p 1$ expression by $\beta$-adrenergic and nuclear receptor agonists [9]. Sympathetic stimulation increases Ucpl expression via protein kinase A-activated binding of cyclic AMP response element binding protein (CREB) to four CREs in the Ucpl enhancer and promoter regions [10]. The enhancer region also appears to be responsible for tissue-specific expression of Ucpl [11] through the interaction between PRDM16, peroxisome proliferator activated receptor gamma coactivator 1alpha $(\mathrm{PGC}-1 \alpha)$ and peroxisome proliferator activated receptor gamma (PPAR $\gamma$ ) acting at the peroxisome proliferator activated receptor response element (PPARE) [12], brown fat regulatory element and the nuclear factor erythroid response element (NF-E2) sites [9].

Methylation of discrete CpGs can modulate the binding of important transcription factors, thereby altering gene expression, including regulating tissue specificity (e.g. leptin and glucose transporter 4 [GLUT4]) [13-16]. Previous studies have demonstrated that the consensus CRE motif which contains a $\mathrm{CpG}$ dinucleotide is a target for DNA methylation and suppresses gene expression [1719]. Furthermore, the nuclear hormone corepressor, RIP140, increases the assembly of histone methyltransferases on the Ucpl promoter leading to the methylation of specific CpGs, whereas increased active histone acetylation and decreased repressive histone marks have been reported in Rip140 (nuclear receptor-interacting protein 1, also known as Nrip1) ${ }^{-/-}$mouse embryonic fibroblasts [20].

Adrenergic stimulation and cold stress in rodents increase energy expenditure by stimulating expression of Ucpl in the interscapular BAT depot during the recruitment of BAT in this depot [21] and by the appearance of brown adipocytes in white adipose tissue (WAT) [8, 22, 23]. The mechanisms underlying the tissue-specific expression of Ucpl expression are currently unknown. Here we demonstrate that $\mathrm{CpG}$ dinucleotide methylation differs between tissues and together with histone modification is involved in the regulation of Ucpl gene expression.

\section{Methods}

Animal experiments Two groups of C57BL/6 mice (Harlan, Loughborough, UK), each consisting of four females, were housed individually in cages measuring $48 \times 15 \times 13 \mathrm{~cm}$ with a $16 \mathrm{~h}$ light and $8 \mathrm{~h}$ dark cycle with access to bedding 
material. All groups had access ad libitum to a standard mouse chow diet. Mouse weight and feed consumption were measured at $24 \mathrm{~h}$ intervals. One group was kept at 22 $\pm 2^{\circ} \mathrm{C}$ for $72 \mathrm{~h}$ (control warm-acclimatised group). A second group was kept at $22 \pm 2^{\circ} \mathrm{C}$ for $48 \mathrm{~h}$ followed by $8 \pm 2^{\circ} \mathrm{C}$ for $24 \mathrm{~h}$ and comprised the cold-acclimatised group. All experiments followed institutional guidelines at the University of Aberdeen and those set out for animal care by the UK Home Office. Animals were killed by concussion followed by cervical dislocation following Home Office guidelines.

Cell culture Medium, sera, vitamins solution and antibiotics/ antimycotics were bought from GIBCO BRL (Paisley, UK). 3T3-L1 cells (ECACC, Salisbury, UK) and HIB-1B cells (kindly provided by B. Spiegelman) were maintained in DMEM with $10 \%$ FBS (Invitrogen, Carlsbad, CA, USA) in $5 \% \mathrm{CO}_{2}$. For differentiation, HIB-1B cells were cultured to confluence (day 0 ) and then exposed to the differentiation cocktail ( $0.5 \mathrm{mmol} / 1$ 3-isobutyl-1-methylxanthine, $250 \mathrm{nmol} / 1$ dexamethasone, $170 \mathrm{nmol} / 1$ insulin, $10 \mathrm{nmol} / 1$ $\mathrm{T}_{3}$ ). After $48 \mathrm{~h}$, cells were maintained in medium containing $5 \% \mathrm{FBS}, 170 \mathrm{nmol} / \mathrm{l}$ insulin and $10 \mathrm{nmol} / 1 \mathrm{~T}_{3}$ until day 7 for harvest, and this medium was replaced daily. 3T3-L1 cells were cultured to confluence (day 0 ) and 2 days later, were differentiated as described for HIB-1B cells.

Methylated cytosine mapping Bisulphite conversion of genomic DNA prepared from cells or tissues was carried out essentially as described by Clark et al. [24]. The modified DNA was purified using a desalting column (Promega Wizard DNA Clean-Up system; Promega, Madison, WI, USA) Methylation was quantified by pyrosequencing using Pyro Q-CpG software (Biotage, Charlottesvile, VA, USA) and performed by The Genome Centre, Barts Hospital, London, UK. Primer sequences and descriptions are provided (see Electronic supplementary material [ESM] Table 1), products destined to be pyrosequenced were amplified with 5'-biotin-labelled primers to allow purification before sequencing.

Methylated promoter reporter luciferase assays Ucp1 promoter fragments inserted into pGL3 firefly luciferase reporter vectors [9] were either methylated by incubation with SssI methylase to methylate all $\mathrm{CpG}$ residues or mockmethylated by the addition of nuclease-free water instead of SSSI. Vectors were then purified using a QiaQuick kit (Qiagen, Valencia, CA, USA). Vectors were then transfected into $80 \%$ confluent HIB-1B and 3T3-L1 cells as previously described [4] using $3 \mu \mathrm{l} / \mu \mathrm{g}$ DNA of FuGene6 (Roche, Burgess Hill, UK) or $2 \mu \mathrm{l} / \mu \mathrm{g}$ DNA of Lipofectamine 2000 (Invitrogen) respectively according to the manufacturers' instructions. Thirty-six hours later, cells were treated with forskolin in serum-free conditions, harvested after $12 \mathrm{~h}$, and firefly and Renilla luciferase activities were measured using the Promega Dual-Glo Luciferase Assay System. The activity of firefly luciferase was normalised to that of Renilla luciferase.

Chromatin immunoprecipitation assays Nuclei, prepared as described by Thomas et al. [25] were cross-linked (1\% formaldehyde for $10 \mathrm{~min}$ ), lysed, sonicated and immunoprecipitated with antibodies against TriMethH3K4 (Abcam, Cambridge, UK) and DiMethH3K9 (Abcam) as described previously [5]. Bound and input fractions were analysed by quantitative real-time PCR (qRTPCR) using the primers given in ESM Table 1. For the suppressed mark (DiMethH3K9) a region of the alpha-fetoprotein ( $A f p)$ start site $(-82$ to $+94 \mathrm{bp})$ was chosen as a positive control because this gene has been shown to be silenced in adult mouse tissues [26]. The positive control for the expressed mark (TriMethH3K4) was the start site of the proline-rich Gla protein (PRGP2), which is a transmembrane protein broadly produced in vertebrates across tissues [27]. The amount of Ucpl DNA was normalised to the amount of Prgp2 (also known as Prrg2) or Afp start site DNA in the bound and input fractions, quantified by qRTPCR.

Real-time PCR Total RNA was extracted from cultured cells and tissue by use of TRI reagent (Sigma, Poole, UK). Before RTPCR, samples were treated with DNA-free DNase to remove contaminating genomic or plasmid DNA. Complementary DNA was generated using the cDNA synthesis kit from Qiagen. Quantitative RTPCR was performed using Sybr green (Qiagen) according to the manufacturer's instructions in Rotor Gene 3000 (Corbett Research, Cambridge, UK). The sequences of the primers used for qRTPCR are given in ESM Table 1. Expression levels for all genes were normalised to the internal control; 18S rRNA.

Oligonucleotide binding assay Nuclear extracts for electrophoretic mobility shift assays (EMSA) were prepared in the presence of protease and protein phosphatase inhibitors as described by Karmanlidis et al. [4]. The oligonucleotide spanned the Ucp1-CRE3 regulatory element: CRE3 5' CTCCTCTACAGCGTCACAGAGGGTC3' and CRE2 5' CACTGAACTAGTCGTCACCTTTCCA3' (CRE motif is in italics). Specific binding was established by coincubating with 10-, 25- and 50-fold excess of unlabelled oligonucleotide. The effect of methylation of the CRE motif in CRE3 and CRE2 was established by coincubating with unlabelled methylated CRE3 5'TCTCCTCTACAGC ${ }^{\mathbf{M E}}$ GTCACA GAGGGTC, or methylated CRE2 5'CACTGAACTAGT $\mathbf{C}^{\mathrm{ME}}$ GTCACCTTTCCA $\left(\mathrm{C}^{\mathrm{ME}} \mathrm{G}\right.$ shows the position of the methylated $\mathrm{CpG}$ ). Competing unlabelled oligonucleotides were added $15 \mathrm{~min}$ before the addition of labelled probe. 
Statistical analysis All the data were analysed with either Student's $t$ test or two-way analysis of variance.

\section{Results}

$C p G$ dinucleotide occurrence in the Ucpl enhancer To identify potential regulatory $\mathrm{CpG}$ dinucleotides in the enhancer region of the Ucpl promoter across species a BLAST analysis (NCBI, Bethesda, MD, USA) against the human and rat genomes was performed using a $621 \mathrm{bp}$ fragment surrounding a $221 \mathrm{bp}$ region of the mouse enhancer that had previously been shown to regulate gene expression in mice (Fig. 1). The first objective was to identify whether this region was homologous across species and the second was to identify $\mathrm{CpG}$ dinucleotides of interest and determine if they were also conserved across species. This analysis identified a $94 \mathrm{bp}$ fragment ( $-2510 \mathrm{bp}$ to $-2416 \mathrm{bp})$ of the mouse enhancer that had $86 \%$ homology with the region -3805 bp to $-3711 \mathrm{bp}$ upstream of human Ucp1. BLAST analysis also showed two regions of homology with the rat genome. The first was a $196 \mathrm{bp}$ fragment of the mouse enhancer region ( $-2653 \mathrm{bp}$ to -2457 bp with $83 \%$ homology) and the second a $105 \mathrm{bp}$ fragment of the mouse enhancer region which shared $88 \%$ homology with another region upstream of the rat Ucp 1 gene ( $-400 \mathrm{bp}$ to $-2295 \mathrm{bp}$ ). Figure 1 shows these BLAST analyses combined with the previous alignment published by Rim and Kozak [9]. The conservation of this enhancer during eutherian evolution has recently been reported [28], in particular, the eutherian conservation of the two CREs in the enhancer (designated CRE3 and CRE2 by Rim and Kozak) as well as the thyroxine response element and PPARE sequences were noted (Fig. 1).

Within the enhancer regions several $\mathrm{CpG}$ dinucleotides were identified by observation (Fig. 1) but not a sufficient number to be classed as a $\mathrm{CpG}$ island in any of the species, as confirmed using an EMBOSS CpGPlot [29]. Interestingly, the only $\mathrm{CpG}$ conserved through the three species was present in the highly conserved CRE3. The human BLAST did not reveal further $\mathrm{CpG}$ dinucleotides, although the rat contains a similar quantity of CpGs to the mouse. Six $\mathrm{CpG}$ dinucleotides (numbered 1 to 6) in a $460 \mathrm{bp}$ region of the mouse enhancer ( -2500 to -2726 from the transcription start site) were selected for studying methylation state using bisulphite mapping. $\mathrm{CpG} 1$ and 2 flanked the $5^{\prime}$ end of the Ucplenhancer and CpG 5 and 6 flanked the 3 ' side. CpG 3 and 4 lay within the Ucpl enhancer and were part of CRE3 and CRE2 as shown in Fig. 1. By including $\mathrm{CpGs}$ beyond the recognised mouse Ucpl enhancer it may be possible to determine if the observed changes are on discrete CpGs, as has been identified for
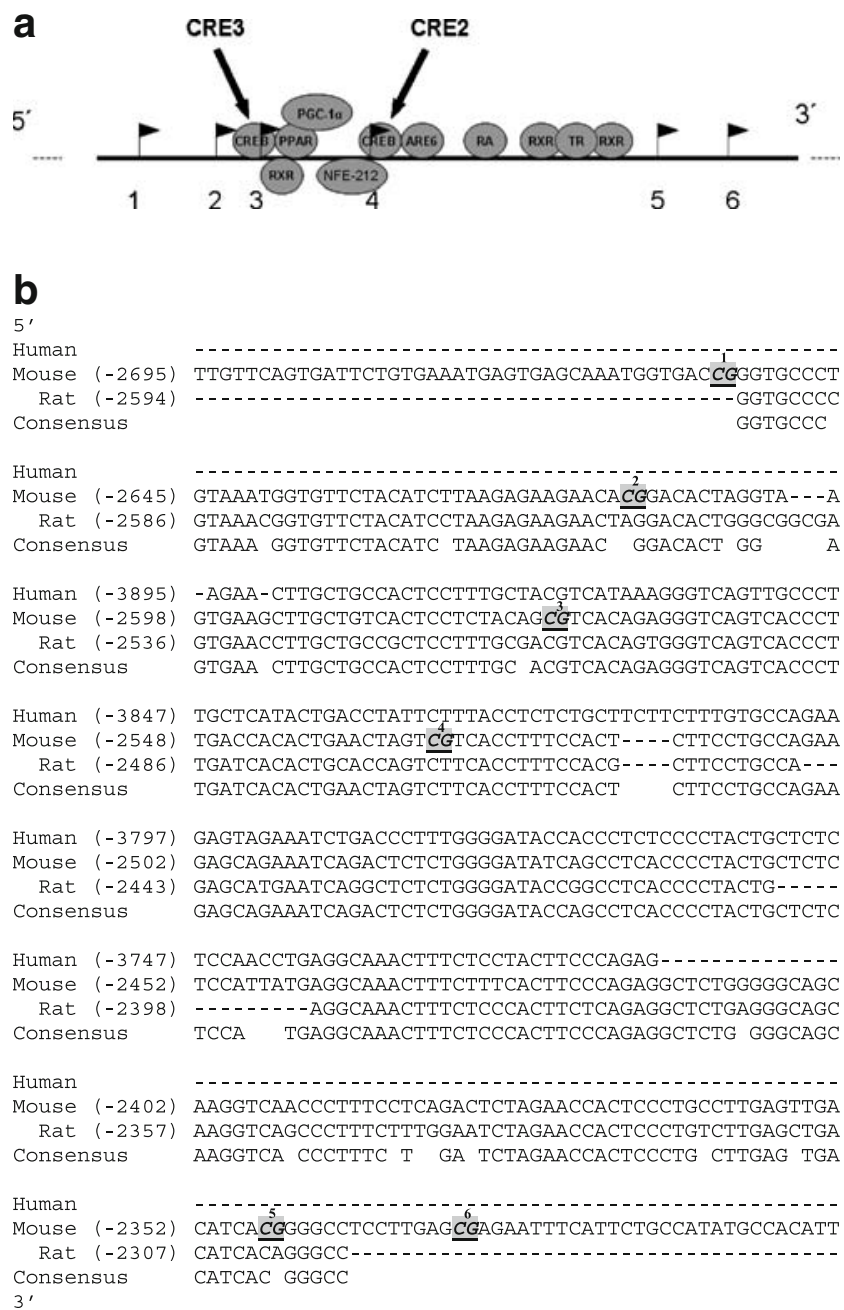

Fig. 1 Location of CpGs and transcription factor binding sites on the Ucpl enhancer. a Selected CpGs of interest indicated by black flags and numbered 1 to 6 relative to cyclic AMP response element (CRE) sites, CRE2 and CRE4. A selection of transcription factors whose binding has been previously described, are shown in their relative positions. CREB, PPAR, PGC- $1 \alpha$, retinoic acid (RA), retinoid X receptor (RXR), thyroid hormone receptor (TR), brown fat response element (BRE) and nuclear factor erythroid derived 212 (NFE-212). b Alignment of mouse, rat and human $350 \mathrm{bp}$ of the Ucpl enhancer. These sequences identified by BLAST are aligned here using Vector NTI. Highlighted grey regions are the core sequences (CGTCA) of the CREs in the mouse promoter with CRE3 positioned upstream of CRE2. CpGs chosen for analysis are underlined and in bold, these are coded 1-6 (5' to $\left.3^{\prime}\right)$ in superscript

genes of GLUT4 and leptin [13, 16], or are occurring over a wider area of DNA.

$C p G$ methylation state in the Ucpl enhancer in adipocyte cell lines We next employed bisulphite mapping to examine the $\mathrm{CpG}$ methylation state of the Ucpl enhancer in brown HIB-1B and white 3T3-L1 adipocytes. Ucp 1 expression was increased $\sim 200$-fold $(p<0.001)$ by treating differentiated HIB-1B cells with forskolin, but the same treatment of 3T3-L1 cells elicited a very much smaller 
increase in Ucp ImRNA levels (Fig. 2a). The methylation state of the six $\mathrm{CpG}$ dinucleotides in the $U c p 1$ enhancer did not differ between undifferentiated and differentiated HIB1B or 3T3-L1 cells and there was no effect of forskolin on the methylation of these CpGs in either cell type (results not shown). The pattern of methylation differed between $\mathrm{CpG}$ dinucleotide position across the Ucpl enhancer of HIB-1B cells, with the methylation state at $\mathrm{CpG}$ positions 3 and 4 lower $(p<0.05)$ than at the other $\mathrm{CpG}$ dinucleotides (Fig. 2b). Surprisingly, 3T3-L1 cells had significantly lower methylation levels than HIB-1B cells at $\mathrm{CpG} 1, \mathrm{CpG} 4$ and CpG $6(p<0.05)$ (Fig. 2b). In contrast, CpG 3 was more methylated in 3T3-L1 cells than in HIB-1B cells $(p<0.001)$ but there was no significant difference in methylation at CpG 5.

To assess the functional importance of methylation state on Ucpl expression we next examined the effects of 5-
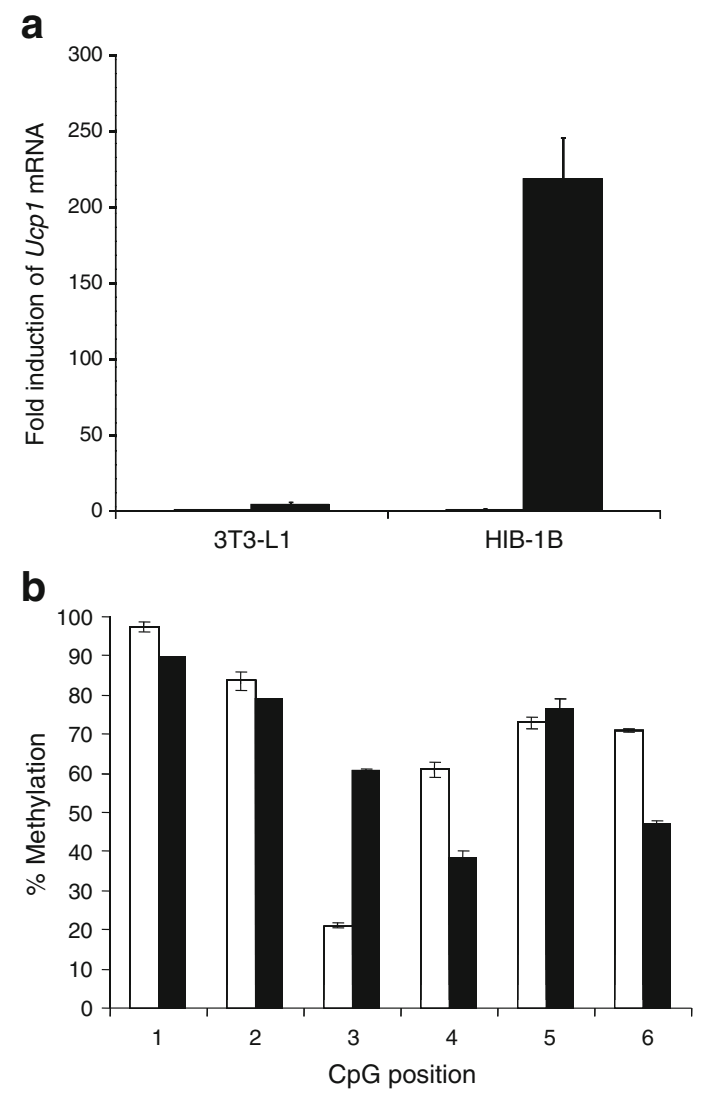

Fig. 2 Ucp 1 mRNA expression and $\mathrm{CpG}$ dinucleotide methylation of the $U c p 1$ enhancer in differentiated HIB-1B and 3T3-L1 cells. a Effect of vehicle (white bar) and $10 \mu \mathrm{mol} / 1$ forskolin (black bar) on Ucp 1 mRNA abundance normalised to $18 \mathrm{~S}$ rRNA. b DNA was extracted from differentiated HIB-1B (white bars) and 3T3-L1 cells (black bars), bisulphite modified, amplified by PCR and pyrosequenced to determine $\mathrm{CpG}$ methylation over positions $1-6$ of the Ucp 1 enhancer. Bars represent mean $\pm \operatorname{SEM}(n=3$ experiments). Significantly different from control, $* * p<0.01$; significant difference between adipose tissues types, ${ }^{*} p<0.05$ aza-deoxycytidine (a methyl transferase inhibitor) which demethylates $\mathrm{CpG}$ dinucleotides on Ucpl methylation and gene expression. Preincubation of 3T3-L1 cells with 5-aza-deoxycytidine significantly reduced DNA methylation by $20-50 \%$ at all $\mathrm{CpG}$ dinucleotides except at position 4 in the $U c p 1$ enhancer (Fig. 3a) and increased both control and forskolin-stimulated Ucp 1 expression. In HIB-1B cells preincubation with 5-aza-deoxycytidine increased basal Ucpl expression (Fig. 3b) and inhibited forskolindependent induction of $U c p 1$ expression.

We next examined the effect on transcriptional activity of methylating $\mathrm{CpG}$ dinucleotides in the $U c p 1$ promoter by treating with the DNA methyltransferase, SssI, a series of different promoter fragments inserted into pGL3 luciferase reporter plasmids, and then transfected into HIB-1B or 3T3L1 cells. Successful methylation of the vectors was confirmed by subsequent digestion with a methylation sensitive restriction enzyme (HpaII) and controls were mock-methylated in the absence of SssI. Figure 4a,b shows the four Ucpl promoter constructs that were examined:

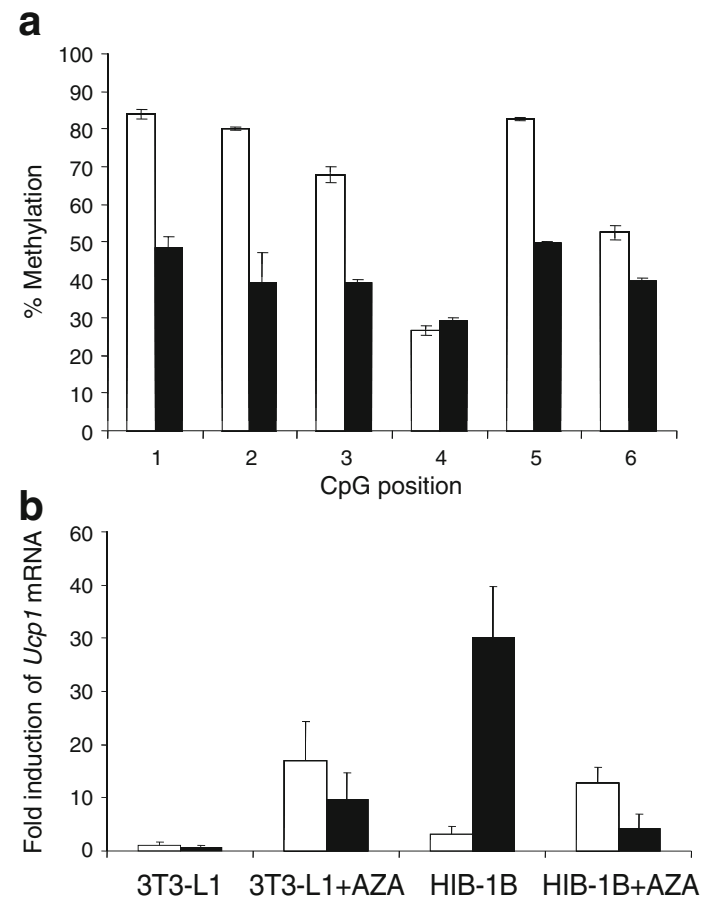

Fig. 3 Effect of 5-aza-deoxycytidine on $\mathrm{CpG}$ dinucleotide methylation of the Ucp1 enhancer and Ucp1 mRNA expression in HIB-1B and 3T3-L1 cells. a $\mathrm{CpG}$ methylation over positions 1 to 6 of the Ucp 1 enhancer in 3T3-L1 cells grown to $80 \%$ confluence and treated with vehicle alone (white bars) or $1 \mu \mathrm{mol} / 15$-aza-deoxycytidine (black bar) for $48 \mathrm{~h}$. b Ucp 1 mRNA transcription normalised to $18 \mathrm{~S}$ rRNA in 3T3-L1 cells and HIB-1B cells treated with $1 \mu \mathrm{mol} / 1$ 5-azadeoxycytidine or vehicle alone for $48 \mathrm{~h}$ before treatment with $10 \mu \mathrm{mol} / 1$ forskolin (black bars) or vehicle (white bars). The data are presented as fold increase in Ucp 1 mRNA over vehicle only 3T3-L1 cells. Bars represent mean \pm SEM ( $n=3$ experiments) 
construct 1 is the empty vector; construct 2 contains the proximal promoter and CRE 4; construct 3 contains both the proximal promoter and the enhancer (which contains CRE 1, 2 and 3); construct 4 contains the entire $3.1 \mathrm{~kb}$ Ucpl promoter. When transfected into HIB-1B cells, transcription from construct 3 (enhancer plus proximal promoter) showed the highest transcriptional activity with lower activities in constructs 2 (proximal promoter) and 4 (3.1 kb Ucp1 promoter), compared with the control mockmethylated empty vector (construct 1). Addition of forskolin stimulated $(p<0.01)$ the transcriptional activity of all Ucp1 mock-methylated promoter constructs. SssI methylation downregulated $(p<0.001)$ the transcriptional activity of all constructs to basal values. When the constructs were transfected into 3T3-L1 cells (Fig. 4b), the transcriptional activity was reduced $(p<0.001)$ by more than half of that observed in HIB-1B cells and methylation of the luciferase vector again decreased activity to basal levels.

Effect of cold stress on CpG methylation state in the Ucp1 enhancer in vivo The studies in adipocyte cell lines clearly demonstrated a role for $\mathrm{CpG}$ methylation in the control of Ucpl transcription so we next determined whether there were any in vivo differences in the methylation state of the
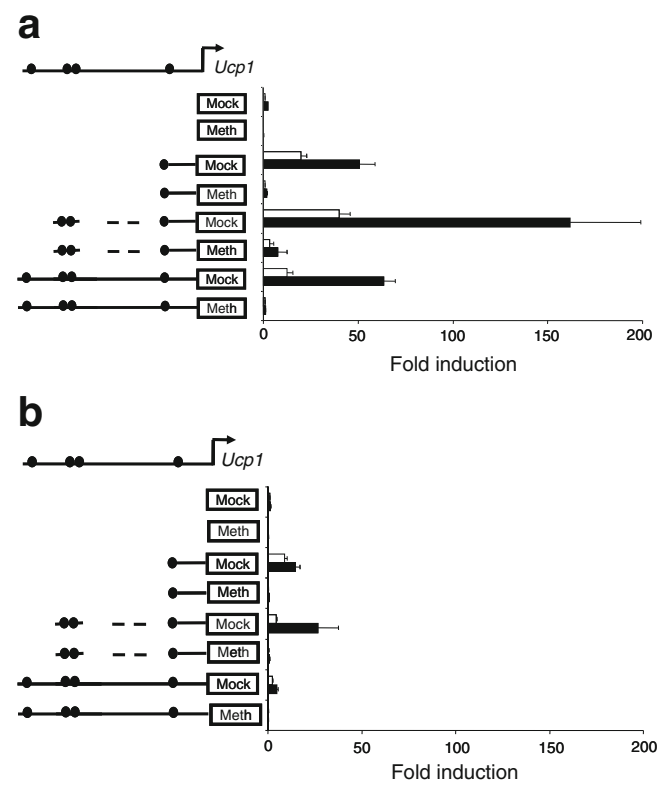

Fig. 4 Effect of methylation of pGL3 luciferase reporter constructs containing various fragments of the Ucpl promoter and transfected into HIB1B and 3T3-L1 cells. a Fold expression of SSSI methylated (Meth) and mock-methylated (Mock) reporter constructs in HIB-1B cells treated with vehicle (white bars) and $10 \mu \mathrm{mol} / \mathrm{l}$ forskolin (black bars). Black circles represent CREs 1, 3, 2 and 4, in order left to right, and the dashed line indicates missing promoter sequences between enhancer and proximal promoter. b Fold expression of SssI methylated (Meth) and mock-methylated (Mock) reporter constructs in 3T3L1 cells treated with vehicle (white bars) and $10 \mathrm{~mol} / \mathrm{l}$ forskolin (black bars). Bars represent mean \pm SEM ( $n=3$ experiments) six $\mathrm{CpG}$ dinucleotides in the Ucplenhancer region between brown and white adipose tissues in mice exposed to a cold environment. Bisulphite mapping of the Ucpl enhancer region was performed on DNA isolated from four tissues (intrascapular BAT [iBAT]; intrascapular WAT [iWAT]; gonadal WAT [gWAT] and liver) and then analysed by bisulphite mapping and pyrosequencing. The iBAT and iWAT were separated by dissection. Ucpl expression was significantly $(p<0.001)$ higher in iBAT compared with iWAT, and very low but clearly present in gWAT. Cold adaptation significantly $(p<0.001)$ increased Ucp1 mRNA four- to sixfold in all adipose tissues sampled and no expression was observed in the liver, which acted as a control non-Ucpl-expressing tissue (Fig. 5a).

There was no effect of cold adaptation on the percentage of $\mathrm{CpG}$ dinucleotide methylation in any of the adipose tissues measured but methylation differed considerably between tissue and $\mathrm{CpG}$ position (Fig 5b,c). At $\mathrm{CpG}$ dinucleotide positions 1-4, iBAT exhibited the lowest methylation state, liver was consistently the highest, and iWAT and gWAT were intermediate $(p<0.01)$. Methylation at $\mathrm{CpG}$ dinucleotide position 5 and 6 , in warm-acclimatised mice, was similar in all tissues (Fig. 5c) but in coldacclimatised mice, methylation in liver significantly $(p<$ 0.01 ) increased to $92 \%$, although there was no change in the adipose tissues. The two white adipose tissue depots (gWAT and iWAT) showed very similar methylation patterns at all positions despite the higher levels of Ucpl expression in iWAT. In the adipose tissues, methylation was significantly lower in $\mathrm{CpG}$ dinucleotide positions 3 and 4, compared with $1,2,5$ and $6(p<0.05)$.

Since $\mathrm{CpG}$ at positions 3 and 4 were within CRE3 and CRE2, respectively, we next established whether methylation of these CpGs in a short double-stranded oligonucleotide with sequences flanking either CRE3 or CRE2, influenced binding of nuclear protein by EMSA. Methylation of the $\mathrm{CpG}$ in CRE2 markedly decreased the competition of excess oligonucleotide with nuclear proteins prepared from brown adipocytes (HIB-1B), to bind to the labelled CRE2 unmethylated oligonucleotide (Fig. 6). In contrast, methylation of the $\mathrm{CpG}$ in the CRE3 oligonucleotide had no effect on competition with nuclear proteins, suggesting that the methylation of the $\mathrm{CpG}$ at position 4, within the CRE2 sequence, may be of functional significance.

We next employed chromatin immunoprecipitation (ChIP) assays to establish whether the Ucpl promoter region was associated with histone marks, indicating either active (TriMethH3K4) or repressed (DiMethH3K9) chromatin states. The start of Ucp 1 transcription was examined because the TriMethH3K4 modification is found around the start of transcription of actively transcribed genes and diminishes moving upstream and downstream from this 

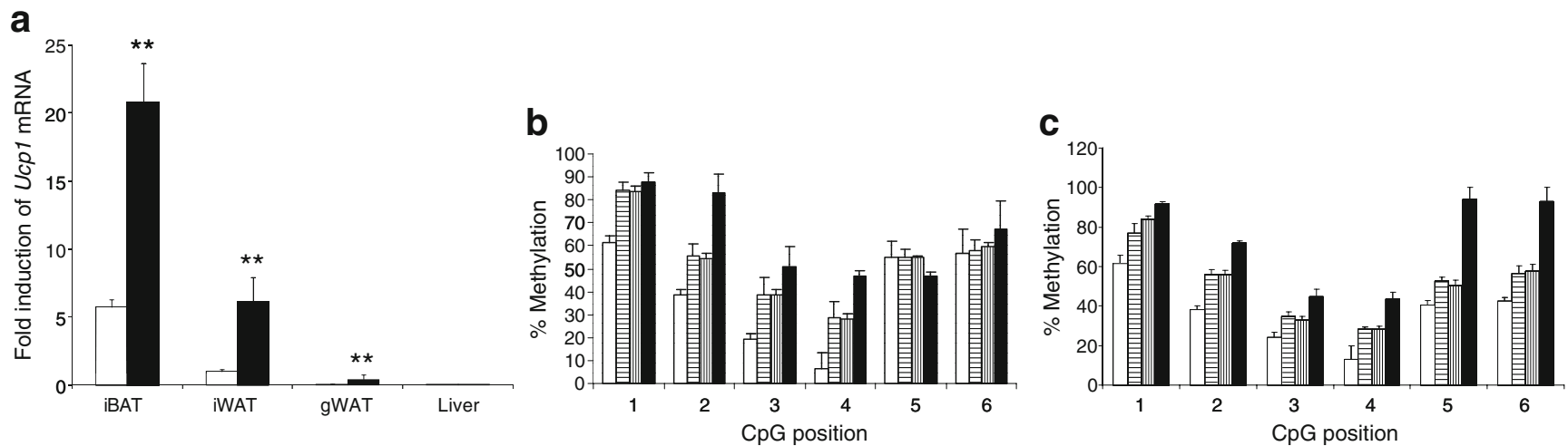

Fig. 5 Effect of cold stress on expression of Ucpl mRNA and methylation of $\mathrm{CpG}$ dinucleotides in the $U c p 1$ enhancer in different tissues from mice. a Ucpl mRNA abundance normalised to $18 \mathrm{~S}$ rRNA in iBAT, iWAT, gWAT and liver from mice housed in either warm $\left(22 \pm 2^{\circ} \mathrm{C}\right.$, white bars $)$ or cold $\left(8 \pm 2^{\circ} \mathrm{C}\right.$, black bars) conditions for $24 \mathrm{~h}$ before sampling. b Methylation of $\mathrm{CpG}$ dinucleotides in positions 1 to 6 of the Ucpl enhancer in tissues from mice housed

point [30]. In both iBAT and gWAT of warm-acclimatised mice, enrichment of the Ucp1 promoter with this mark was low relative to the control gene, Prgp2 (Fig. 7a). In the cold-adapted group the Ucp 1 promoter remained depleted in TriMethH3K4 immunoprecipitates in gWAT but showed great enrichment $(p<0.01)$ in BAT, suggesting that the promoter became associated with active chromatin. This result corresponded to the large increase in $U c p 1$ mRNA

Probe
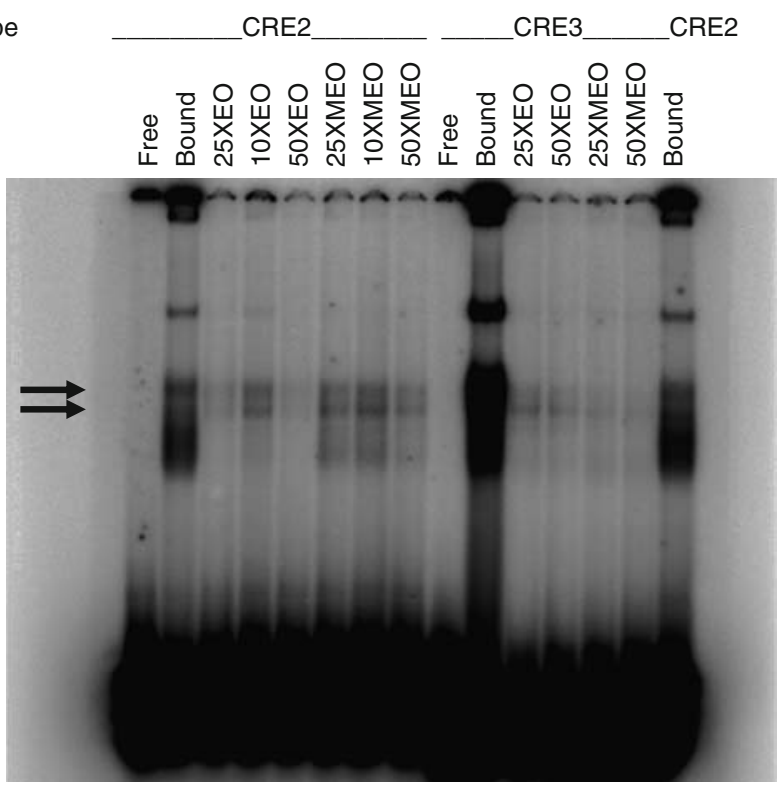

Fig. 6 Effect of methylation of the $\mathrm{CpG}$ in CRE2 and CRE3 sequences on binding to nuclear proteins. End-labelled oligonucleotide probes containing sequences flanking either CRE2 or CRE3 were incubated in the absence (free) and presence (bound) of nuclear proteins prepared from HIB-1B cells. Competition of each probe with oligonucleotide (EO) or excess methylated oligonucleotide (MEO) was examined at $10 \times, 25 \times$ and $50 \times$ the probe concentration, as indicated, in the presence of nuclear proteins in a warm environment. c Methylation of $\mathrm{CpG}$ dinucleotides in positions 1 to 6 of the $U c p 1$ enhancer in tissues from mice housed in a cold environment: iBAT (black bars), iWAT (white bars), gWAT (bars with horizontal stripes) and liver (bars with vertical stripes). Bars represent mean \pm SEM ( $n=4$ mice). Significant difference between warm and cold, ${ }^{* *} p<0.01$

expression in iBAT in cold-acclimatised mice. When the chromatin was immunoprecipitated with an antibody against the repressed DiMethH3K9 mark (Fig. 7b), the Ucp1 promoter was depleted of this mark in nuclei from both types of adipose tissue relative to the repressed control gene, Afp start site. Association of the repressed DiMethH3K9 mark with the Ucp1 enhancer region relative to Afp (Fig. 7c) demonstrated that gWAT was enriched compared with iBAT, although this was only significantly different $(p<0.05)$ in the cold-adapted mice. Taken together the ChIP assay results suggest that the Ucpl promoter is associated with an active and repressed chromatin state in iBAT and gWAT, respectively, and that cold induces a more active chromatin state around the $U c p 1$ promoter start site, in line with a rise in $U c p 1$ expression.

\section{Discussion}

In this study we demonstrate that the methylation state of CpG dinucleotides in the Ucpl enhancer exhibits positionspecific and adipose tissue-specific patterns in both cell lines and murine adipose tissue and that promoter methylation has functional importance in the regulation of Ucpl expression.

$\mathrm{CpG}$ dinucleotide methylation state in the Ucp 1 enhancer was not altered during either differentiation of white 3T3-L1 and brown HIB-1B pre-adipocytes or treatment with forskolin, despite increased Ucpl expression, Surprisingly, methylation state was higher at $\mathrm{CpG}$ dinucleotide positions 1,4 and 6 in HIB-1B compared with 3T3-L1 cells, and only clearly lower in HIB-1B cells at position 3 . Similar analysis of tissue samples taken in vivo demon- 


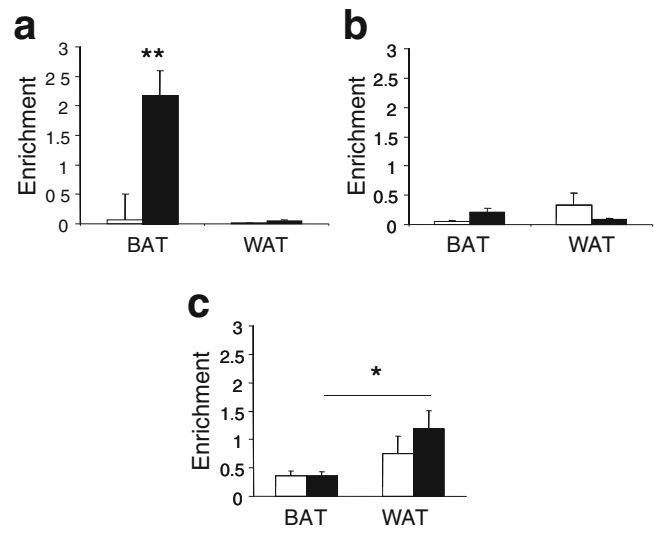

Fig. 7 Chromatin immunoprecipitation analysis of the association of the Ucpl promoter with active (TriMethH3K4) and repressed (DiMethH3K9) histone marks. a Immunoprecipitation by TriMethH3K4 of the Ucp 1 promoter close to the transcription start site, relative to the promoter of the expressed reference gene Prgp2. b Immunoprecipitation by DiMethH3K9 of the Ucp 1 promoter close to the transcription start site, relative to the promoter of the suppressed gene $A f p$. c Immunoprecipitation by DiMethH3K9 of the Ucp 1 enhancer region, relative to the promoter of the suppressed reference gene $A f p$. Adipose tissue nuclei were extracted and cross-linked and the DNA was immunoprecipitated using anti-TriMethH3K4 or antiDiMethH3K9. Ucp1 start site and Prgp2, Afp start site DNA in the bound and input fractions were quantified by qRTPCR. iBAT and gWAT were taken from mice housed in either warm $\left(22 \pm 2^{\circ} \mathrm{C}\right.$, white bars) or cold, $\left(8 \pm 2^{\circ} \mathrm{C}\right.$, black bars) conditions for $24 \mathrm{~h}$ before sampling. Bars represent the mean \pm SEM of triplicate assays

strated that methylation state was consistently lower in BAT compared with WAT and liver, at $\mathrm{CpG}$ dinucleotide positions 1-4 in the Ucpl enhancer. Furthermore, the methylation state of $\mathrm{CpG}$ dinucleotides at positions 3 and 4, in the CRE3 and CRE2 sequences, respectively, were the lowest in all tissues, with position 4 within CRE2 being less than $10 \%$. Binding of a labelled oligonucleotide probe containing CRE2 and flanking sequences, to brown adipocyte nuclear proteins, was displaced in a concentration-dependent manner by excess unlabelled probe but not when the excess unlabelled probe was methylated at $\mathrm{CpG}$ dinucleotide position 4 . A similar study examining a labelled CRE3 oligonucleotide probe failed to observe any decrease in competition with excess unlabelled methylated probe. We have previously demonstrated using EMSA and ChIP that HIB-1B nuclear extracts contain bZip transcription factors, including CREB, which bind to CREs $[4,5]$, therefore suggesting that methylation of the $\mathrm{CpG}$ dinucleotide in the CRE2 sequence, decreases binding of transcription factors.

Further functional evidence that $U c p 1$ promoter methylation is linked to gene expression was obtained by treatment with 5-aza-deoxycytidine in 3T3-L1 cells. 5Aza-deoxycytidine treatment decreased methylation of the Ucp 1 enhancer in 3T3-L1 cells and significantly increased expression of basal and forskolin-stimulated Ucpl expres- sion. Furthermore, methylation of luciferase reporter plasmids containing the whole $3.1 \mathrm{~kb}$, or fragments of the Ucp 1 promoter, before transfection into HIB-1B or 3T3-L1 cells, inhibited transcriptional activity from the promoter. This effect was greatest for reporter vectors containing just the enhancer and promoter sequences where the CREs are located, providing strong evidence that methylation of $\mathrm{CpG}$ dinucleotides in CREs in the Ucpl promoter, inhibits transcription. Similar evidence for the role of methylation in the control of other genes has been reported [13, 31-36].

Global demethylation of the HIB-1B cells with 5-azadeoxycytidine unexpectedly inhibited forskolin-induced $U c p 1$ expression, suggesting a role for methylation state of unidentified factors that inhibit brown adipogenesis. Treatment of mouse Swiss 3T3 fibroblasts with 5-azadeoxycytidine has previously been shown to direct this cell line to the myogenic lineage [37]. Lineage tracing studies have recently discovered that BAT shares a common cell progenitor with muscle and not WAT depot cells, as previously thought [38]. Downregulation of the BATspecific gene Ucp1 in HIB-1B cells, but not in 3T3-L1 cells, by 5 -aza-deoxycytidine may therefore be the result of commitment to a myogenic lineage in the brown adipocyte cell line.

Previous studies have demonstrated that methylation of CpG dinucleotides in CREs inhibits the binding of CREB and transcriptional responses to cAMP $[19,36]$. CRE3 and CRE2 have previously been shown to bind CREB and are essential for the maximal response of the gene to adrenergic stimulation [9]. However, the lower $\mathrm{CpG}$ methylation states in these CREs were not altered by either short-term ( $24 \mathrm{~h})$ exposure to cold in vivo or treatment of adipocyte cell lines with forskolin, suggesting that methylation state does not play a role in acute adrenergic-stimulated Ucpl expression. These two CpGs may be held in a lower state of methylation to allow response to stimuli because they are also 3-15 bases from the PPARE, brown fat regulatory element and NF-E2 response element, all of which have been implicated in the control of brown adipose tissue differentiation $[9,10]$. Interestingly, the $\mathrm{CpG}$ dinucleotide in position 3 was the only example of methylation state being lower in HIB-1B, the Ucp 1-expressing cell line, compared with 3T3-L1 cells.

The ChIP studies demonstrated that the amount of the active TriMethH3K4 mark bound to the proximal Ucp1 promoter increased in iBAT but not in gWAT following cold exposure. Conversely, the Ucpl promoter in gWAT was more enriched with the repressed DiMethH3K4 histone mark, compared with in iBAT. These results agree with those of Kiskinis et al. [20] suggesting that the control of Ucpl expression involves modification of histone tails associated with the Ucpl locus. Enrichment of the enhancer with the repressed histone mark was greater in gWAT than 
the proximal promoter region, suggesting that histone modification may differ across the $3.1 \mathrm{~kb}$ Ucpl 5' regulatory region.

In conclusion, we demonstrate that $\mathrm{CpG}$ methylation of the Ucpl enhancer exhibits CpG-position and tissuespecific patterns and, using cell lines, that this difference may have functional importance suggesting that the regulation of Ucpl expression involves chromatin modification. The results identify chromatin remodelling as a plausible target for future research to identify mechanisms to upregulate $U c p 1$ expression in adipocytes in the search for novel treatments of obesity.

Acknowledgements We thank A. Thorne (University of Portsmouth) for his advice and Biotechnology and Biological Sciences Research Council (BBSRC) who funded the project.

Duality of interest The authors declare that there is no duality of interest associated with this manuscript.

Open Access This article is distributed under the terms of the Creative Commons Attribution Noncommercial License which permits any noncommercial use, distribution, and reproduction in any medium, provided the original author(s) and source are credited.

\section{References}

1. Arch JR (2002) Beta(3)-adrenoceptor agonists: potential, pitfalls and progress. Eur J Pharmacol 440:99-107

2. Nedergaard J, Bengtsson T, Cannon B (2007) Unexpected evidence for active brown adipose tissue in adult humans. Am J Physiol Endocrinol Metab 293:E444-452

3. Tiraby C, Tavernier G, Lefort C et al (2003) Acquirement of brown fat cell features by human white adipocytes. J Biol Chem 278:33370-33376

4. Karamanlidis G, Karamitri A, Docherty K, Hazlerigg DG, Lomax MA (2007) C/EBPbeta reprograms white 3T3-L1 preadipocytes to a brown adipocyte pattern of gene expression. J Biol Chem 282:24660-24669

5. Karamitri A, Shore AM, Docherty K, Speakman JR, Lomax MA (2009) Combinatorial transcription factor regulation of the cyclic AMP-response element on the Pgc-1alpha promoter in white 3T3L1 and brown HIB-1B preadipocytes. J Biol Chem 284:2073820752

6. Lomax MA, Sadiq F, Karamanlidis G, Karamitri A, Trayhurn P, Hazlerigg DG (2007) Ontogenic loss of brown adipose tissue sensitivity to beta-adrenergic stimulation in the ovine. Endocrinology 148:461-468

7. Cavalli G (2006) Chromatin and epigenetics in development: blending cellular memory with cell fate plasticity. Development 133:2089-2094

8. Cannon B, Nedergaard J (2004) Brown adipose tissue: function and physiological significance. Physiol Rev 84:277-359

9. Rim JS, Kozak LP (2002) Regulatory motifs for CREB-binding protein and $\mathrm{Nfe} 212$ transcription factors in the upstream enhancer of the mitochondrial uncoupling protein 1 gene. J Biol Chem 277:34589-34600

10. Kozak UC, Kopecky J, Teisinger J, Enerback S, Boyer B, Kozak LP (1994) An upstream enhancer regulating brown-fat-specific expression of the mitochondrial uncoupling protein gene. Mol Cell Biol 14:59-67

11. Cassard-Doulcier AM, Gelly C, Bouillaud F, Ricquier D (1998) A 211-bp enhancer of the rat uncoupling protein-1 (UCP-1) gene controls specific and regulated expression in brown adipose tissue. Biochem J 333(Pt 2):243-246

12. Seale P, Kajimura S, Spiegelman BM (2009) Transcriptional control of brown adipocyte development and physiological function - of mice and men. Genes Dev 23:788-797

13. Melzner I, Scott V, Dorsch K et al (2002) Leptin gene expression in human preadipocytes is switched on by maturation-induced demethylation of distinct CpGs in its proximal promoter. J Biol Chem 277:45420-45427

14. Stoger R (2006) In vivo methylation patterns of the leptin promoter in human and mouse. Epigenetics 1:155-162

15. Yokomori N, Tawata M, Onaya T (1999) DNA demethylation during the differentiation of 3T3-L1 cells affects the expression of the mouse GLUT4 gene. Diabetes 48:685-690

16. Yokomori N, Tawata M, Onaya T (2002) DNA demethylation modulates mouse leptin promoter activity during the differentiation of 3T3-L1 cells. Diabetologia 45:140-148

17. Mancini DN, Rodenhiser DI, Ainsworth PJ et al (1998) CpG methylation within the $5^{\prime}$ regulatory region of the BRCA1 gene is tumor specific and includes a putative CREB binding site. Oncogene 16:1161-1169

18. Kroft TL, Jethanandani P, McLean DJ, Goldberg E (2001) Methylation of $\mathrm{CpG}$ dinucleotides alters binding and silences testis-specific transcription directed by the mouse lactate dehydrogenase C promoter. Biol Reprod 65:1522-1527

19. Demura M, Bulun SE (2008) CpG dinucleotide methylation of the CYP19 I.3/II promoter modulates cAMP-stimulated aromatase activity. Mol Cell Endocrinol 283:127-132

20. Kiskinis E, Hallberg M, Christian M et al (2007) RIP140 directs histone and DNA methylation to silence $U c p 1$ expression in white adipocytes. EMBO J 26:4831-4840

21. Cousin B, Bascands-Viguerie N, Kassis N et al (1996) Cellular changes during cold acclimatation in adipose tissues. J Cell Physiol 167:285-289

22. Cousin B, Cinti S, Morroni M et al (1992) Occurrence of brown adipocytes in rat white adipose tissue: molecular and morphological characterization. J Cell Sci 103(Pt 4):931-942

23. Guerra C, Koza RA, Yamashita H, Walsh K, Kozak LP (1998) Emergence of brown adipocytes in white fat in mice is under genetic control. Effects on body weight and adiposity. J Clin Invest 102:412-420

24. Clark SJ, Harrison J, Paul CL, Frommer M (1994) High sensitivity mapping of methylated cytosines. Nucleic Acids Res 22:2990-2997

25. Thomas J (1998) Isolation and fractionation of chromatin linker histones. In: Gould H (ed) Chromatin. Oxford University Press, Oxford, pp 1-34

26. Nguyen TT, Cho K, Stratton SA, Barton MC (2005) Transcription factor interactions and chromatin modifications associated with p53-mediated, developmental repression of the alpha-fetoprotein gene. Mol Cell Biol 25:2147-2157

27. Kulman JD, Harris JE, Xie L, Davie EW (2007) Proline-rich Gla protein 2 is a cell-surface vitamin K-dependent protein that binds to the transcriptional coactivator Yes-associated protein. Proc Natl Acad Sci USA 104:8767-8772

28. Jastroch M, Withers KW, Taudien S et al (2008) Marsupial uncoupling protein 1 sheds light on the evolution of mammalian nonshivering thermogenesis. Physiol Genomics 32:161-169

29. Larsen F, Gundersen G, Lopez R, Prydz H (1992) CpG islands as gene markers in the human genome. Genomics 13:1095-1107

30. Barski A, Cuddapah S, Cui K et al (2007) High-resolution profiling of histone methylations in the human genome. Cell 129:823-837 
31. Christman JK (2002) 5-Azacytidine and 5-aza-2'-deoxycytidine as inhibitors of DNA methylation: mechanistic studies and their implications for cancer therapy. Oncogene 21:5483-5495

32. Stresemann C, Lyko F (2008) Modes of action of the DNA methyltransferase inhibitors azacytidine and decitabine. Int $\mathrm{J}$ Cancer 123:8-13

33. Wabitsch M, Bruderlein S, Melzner I, Braun M, Mechtersheimer G, Moller P (2000) LiSa-2, a novel human liposarcoma cell line with a high capacity for terminal adipose differentiation. Int $\mathrm{J}$ Cancer 88:889-894

34. Yoshida M, Horinouchi S (1999) Trichostatin and leptomycin. Inhibition of histone deacetylation and signal-dependent nuclear export. Ann NY Acad Sci 886:23-36
35. Yoshida M, Horinouchi S, Beppu T (1995) Trichostatin A and trapoxin: novel chemical probes for the role of histone acetylation in chromatin structure and function. Bioessays $17: 423-430$

36. Zhang X, Odom DT, Koo SH et al (2005) Genome-wide analysis of cAMP-response element binding protein occupancy, phosphorylation, and target gene activation in human tissues. Proc Natl Acad Sci USA 102:4459-4464

37. Taylor SM, Jones PA (1979) Multiple new phenotypes induced in $10 \mathrm{~T} 1 / 2$ and $3 \mathrm{~T} 3$ cells treated with 5-azacytidine. Cell 17:771-779

38. Seale P, Bjork B, Yang W et al (2008) PRDM16 controls a brown fat/skeletal muscle switch. Nature 454:961-967 